\title{
Cyclic DNA codes over the ring $\mathbb{Z}_{4}+u \mathbb{Z}_{4}+u^{2} \mathbb{Z}_{4}$
}

Research Article

\author{
Karthick Gowdhaman, Somi Gupta, Cruz Mohan, Kenza Guenda, Durairajan \\ Chinnapillai
}

\begin{abstract}
In this work, we have investigated the one generator cyclic DNA codes with reverse and reverse complement constraints over the ring $R=\mathbb{Z}_{4}+u \mathbb{Z}_{4}+u^{2} \mathbb{Z}_{4}$ with $u^{3}=0$. Skew cyclic codes with reverse complement constraint are constructed over $R$. We have also determined a one-to-one correspondence between the elements of the ring $R$ and DNA codons satisfying the Watson-Crick complement. Finally, we have established some examples which satisfy the given constraints.
\end{abstract}

2010 MSC: 94B05, 94B15

Keywords: DNA codes, Skew codes, Reversible codes

\section{Introduction}

DNA is shortened word of Deoxyribonucleic acid. It is a molecule made of two chains that curl around one another to frame a two-fold helix conveying the hereditary guidelines utilized in the development of all creatures. This two-fold helix is assembled by blending the four fundamental structure units $A$ (ADENINE), $C$-(CYTOSINE), $G$-(GUANINE) and $T$-(THYMINE) which are called the nucleotides held by Hydrogen ties. The DNA strand is held by an important feature called complementary base pairing which connects the Watson-Crick complementary bases with each other denoted by $\bar{A}=T, \bar{G}=C, \bar{C}=$ $G, \bar{T}=A$.

In [4] Adleman performed a successful experiment for DNA computing. The basic idea of his work was to use DNA which is an ideal source of computing due to its dense and self-replicating property to solve a mathematical problem. After this successful experiment, the area of DNA computing was flooded with different approaches such as DNA tile assembly, the building of DNA nanostructures, DNA-based data storage system and study of error-correcting properties of DNA.

Karthick Gowdhaman, Cruz Mohan, Durairajan Chinnapillai; Department of Mathematics, Bharathidasan University, Tiruchirapalli, Tamil Nadu, India (email: karthimath123@bdu.ac.in, cruzmohan@gmail.com, cdurai66@rediffmail.com).

Somi Gupta; Department of Mathematics and Applications "Renato Caccioppoli", University of Napoli Federico II, Naples, Italy (email: gupta\%somi200@gmail.com).

Kenza Guenda; Laboratory of Algebra and Number Theory, USTHB, Algiers (email: ken.guenda@gmail.com). 
Cyclic codes over rings have been studied by many authors (see for example [6] and [2]). Later DNA cyclic codes have gained interest of many researchers for their applications (see [16], [11], [18], [12], [14], [19], [5]). Abhay et al. [10] have studied DNA cyclic codes over the ring $\mathbb{Z}_{4}+u \mathbb{Z}_{4}$ with $u^{2}=0$. Cyclic codes over the ring $R=\mathbb{Z}_{4}+u \mathbb{Z}_{4}+u^{2} \mathbb{Z}_{4}$ with $u^{3}=0$ have been studied by Ozen et al. in [15]. Cyclic code over skew polynomial ring have been constructed by [7]. Boucher and Ulmer [8] have found a link between arithmetic structure of skew polynomials and existence of such codes. In [17] Siap et al. studied skew cyclic code of arbitrary length and established a strong connection with well known codes. Most recently, the DNA codes over ring of order 256 has been studied in [9]. They have obtained DNA skew cyclic codes over the ring $\mathbb{F}_{2}+u \mathbb{F}_{2}+v \mathbb{F}_{2}+w \mathbb{F}_{2}+u v \mathbb{F}_{2}+u w \mathbb{F}_{2}+v w \mathbb{F}_{2}+u v w \mathbb{F}_{2}$, where, $u^{2}=0, v^{2}=v, w^{2}=w, u v=v u, u w=w u, v w=w v$, addressing reversibility problem.

In this work, we have investigated the one generator cyclic DNA codes with reverse and reverse complement constraint over the ring $R=\mathbb{Z}_{4}+u \mathbb{Z}_{4}+u^{2} \mathbb{Z}_{4}$ with $u^{3}=0$. Skew cyclic codes with reverse complement constraint are constructed over $R$. We have also determined a one-to-one correspondence between elements of the ring $R$ and DNA codons satisfying Watson-Crick complement. Finally, we have established some examples which satisfy the given constraints. Beside the theoretical results concerning the reverse and the reverse complement codes over the ring $R$ and the one-to-one correspondence with codes, our other motivation in choosing this ring is the fact that it contains an additive subgroup of order 16. Then the idea of the additive stem distance characterizing the hybridization energy given in [12] can be extended to this subgroup and then to the ring.

This paper is structured in the following way: Section 2 contains basic definitions of cyclic DNA codes. We have established a one-to-one correspondence between elements of the ring $R=\mathbb{Z}_{4}+u \mathbb{Z}_{4}+u^{2} \mathbb{Z}_{4}$ with $u^{3}=0$, elements of $\mathbb{Z}_{4}^{3}$ and 64 codons. In Section 3 , we have studied the reversibility condition of one generator cyclic codes over the ring $R$ and have determined reverse complement codes. Binary image of the ring and skew cyclic codes are studied in Sections 4 and 5 respectively. In Section 6 , we have obtained some examples. Finally, Section 7 concludes the paper with some establishments of future work that can be done using this work.

\section{Preliminaries}

Ozen et al. in [15] have determined cyclic codes over the ring $R=\mathbb{Z}_{4}+u \mathbb{Z}_{4}+u^{2} \mathbb{Z}_{4}$, where $u^{3}=0$. They obtained that the total number of cyclic codes over $R$ is $13^{r}$. They also found the general form of generator of cyclic codes over $R$ and one generator cyclic codes (cyclic codes generated by one element) over the ring $R$.

Let $R=\mathbb{Z}_{4}+u \mathbb{Z}_{4}+u^{2} \mathbb{Z}_{4}=\left\{a+u b+u^{2} c \mid a, b, c \in \mathbb{Z}_{4}\right\}$, where $u^{3}=0$. The ring $R$ has 11 nontrivial ideals given by

$$
\begin{aligned}
A_{2 u^{2}}= & \left\{0,2 u^{2}\right\} \\
A_{u^{2}}= & \left\{0, u^{2}, 2 u^{2}, 3 u^{2}\right\} \\
A_{2 u}= & \left\{0,2 u, 2 u^{2}, 2 u+2 u^{2}\right\} \\
A_{2 u+u^{2}}= & \left\{0,2 u^{2}, 2 u+u^{2}, 2 u+3 u^{2}\right\} \\
A_{2 u, u^{2}}= & \left\{0, u^{2}, 2 u^{2}, 3 u^{2}, 2 u, 2 u+u^{2}, 2 u+2 u^{2}, 2 u+3 u^{2}\right\} \\
A_{2}= & \left\{0,2,2 u, 2 u^{2}, 2+2 u, 2+2 u^{2}, 2 u+2 u^{2}, 2+2 u+2 u^{2}\right\} \\
A_{2+u^{2}}= & \left\{0,2 u, 2 u^{2}, 2 u+2 u^{2}, 2+u^{2}, 2+3 u^{2}, 2+2 u+u^{2}, 2+2 u+3 u^{2}\right\} \\
A_{2, u^{2}}= & \left\{0,2,2 u, u^{2}, 2 u^{2}, 3 u^{2}, 2+2 u, 2+u^{2}, 2+2 u^{2}, 2+3 u^{2}, 2 u+u^{2}, 2 u+2 u^{2},\right. \\
& \left.2 u+3 u^{2}, 2+2 u+u^{2}, 2+2 u+2 u^{2}, 2+2 u+3 u^{2}\right\}
\end{aligned}
$$




$$
\begin{aligned}
A_{u}= & \left\{0, u, 2 u, 3 u, u^{2}, 2 u^{2}, 3 u^{2}, u+u^{2}, u+2 u^{2}, u+3 u^{2}, 2 u+u^{2}, 2 u+2 u^{2},\right. \\
& \left.2 u+3 u^{2}, 3 u+u^{2}, 3 u+2 u^{2}, 3 u+3 u^{2}\right\} \\
A_{2+u}= & \left\{0,2 u, u^{2}, 2 u^{2}, 3 u^{2}, 2+u, 2+3 u, 2 u+u^{2}, 2 u+2 u^{2}, 2 u+3 u^{2}, 2+u+u^{2},\right. \\
& \left.2+u+2 u^{2}, 2+u+3 u^{2}, 2+3 u+u^{2}, 2+3 u+2 u^{2}, 2+3 u+3 u^{2}\right\} \\
A_{2, u}= & \left\{0,2, u, 2 u, 3 u, u^{2}, 2 u^{2}, 3 u^{2}, 2+u, 2+2 u, 2+3 u, 2+u^{2}, 2+2 u^{2}, 2+3 u^{2},\right. \\
& u+u^{2}, u+2 u^{2}, u+3 u^{2}, 2 u+u^{2}, 2 u+2 u^{2}, 2 u+3 u^{2}, 3 u+u^{2}, 3 u+2 u^{2}, \\
& 3 u+3 u^{2}, 2+u+u^{2}, 2+u+2 u^{2}, 2+u+3 u, 2+2 u+u^{2}, 2+2 u+2 u^{2}, \\
& \left.2+2 u+3 u^{2}, 2+3 u+u^{2}, 2+3 u+2 u^{2}, 2+3 u+3 u^{2}\right\} .
\end{aligned}
$$

The ring $R$ is a finite local ring with $A_{2, u}$ as its unique maximal ideal (as it contains all the non-zero divisors of $R$ ). The residue field of $R$ is $\mathcal{K}=R / A_{2, u}=\left\{0+A_{2, u}, 1+A_{2, u}\right\} \cong \mathbb{Z}_{2}$. Let $p(x)$ be a monic basic irreducible polynomial of degree $m$ in $R[x]$, then the Galois ring extension over $R$ is defined by the residue class ring $Q_{m}=R[x] /(p(x))$ having $64^{m}$ elements.

The triplet of nucleotides called codons is the basic coding unit for amino acids during the protein synthesis in living organisms. Since the ring $R$ has 64 elements, then it is convenient to describe a one-to-one correspondence between the elements of $R$ and the codons. In the following table, we give a one-to-one correspondence between the elements of $R$, the elements of $\mathbb{Z}_{4}^{3}$ and the codons. The oneto-one correspondence, called Gray map between the rings $R$ and $\mathbb{Z}_{4}^{3}$ is defined as $\psi: R \rightarrow \mathbb{Z}_{4}^{3}$ with $\left(a+u b+u^{2} c\right) \mapsto(a, b, c)$, where $a, b, c \in \mathbb{Z}_{4}$. Another important one-to-one correspondence between the elements of $\mathbb{Z}_{4}^{3}$ and $D^{3}$ is denoted by $\phi: \mathbb{Z}_{4}^{3} \rightarrow D^{3}$, where $D$ denotes the set of all nucleotides. The full names of codons have been taken from http://www.hgmd.cf.ac.uk/docs/cd_amino.html.

Table 1. Correspondence between elements of $R, \mathbb{Z}_{4}^{3}$ and Codons

\begin{tabular}{cccc}
\hline$a \in R$ & $\psi(a) \in \mathbb{Z}_{4}^{3}$ & $\phi(a) \in D^{3}$ & Full Name of Codons \\
\hline 0 & $(0,0,0)$ & GTG & Valine \\
$u$ & $(0,1,0)$ & CCG & Alanine \\
$u+u^{2}$ & $(0,1,1)$ & CGA & Arginine \\
$u+2 u^{2}$ & $(0,1,2)$ & CAA & Glutamine \\
$u+3 u^{2}$ & $(0,1,3)$ & TAG & Termination (amber) \\
$2 u+u^{2}$ & $(0,2,1)$ & CAT & Histidine \\
$2 u+2 u^{2}$ & $(0,2,2)$ & CTC & Serine \\
$2 u+3 u^{2}$ & $(0,2,3)$ & ATA & Isoleucine \\
$3 u+u^{2}$ & $(0,3,1)$ & GCG & Alanine \\
$3 u+2 u^{2}$ & $(0,3,2)$ & TCG & Serine \\
$3 u+3 u^{2}$ & $(0,3,3)$ & TAA & Termination (ochre) \\
1 & $(1,0,0)$ & GGC & Arginine \\
$1+u$ & $(1,1,0)$ & TGA & Termination (opal or umber) \\
$1+u+u^{2}$ & $(1,1,1)$ & GGG & Glycine \\
$1+u+2 u^{2}$ & $(1,1,2)$ & GCA & Alanine \\
$1+u+3 u^{2}$ & $(1,1,3)$ & GAA & Glutamate \\
$1+2 u$ & $(1,2,0)$ & CCA & Proline \\
$1+2 u+u^{2}$ & $(1,2,1)$ & TTT & Phenylalanine \\
$1+2 u+2 u^{2}$ & $(1,2,2)$ & CCT & Proline \\
$1+2 u+3 u^{2}$ & $(1,2,3)$ & AGG & Arginine \\
$1+3 u$ & $(1,3,0)$ & TGG & Trytophan \\
$1+3 u+u^{2}$ & $(1,3,1)$ & TTA & Leucine \\
$1+3 u+2 u^{2}$ & $(1,3,2)$ & TAC & Tyrosine \\
$1+3 u+3 u^{2}$ & $(1,3,3)$ & TGC & Cysteine \\
2 & $(2,0,0)$ & GAG & Arginine \\
$2 u$ & $(0,2,0)$ & ACA & Threonine \\
$2+u$ & $(2,1,0)$ & AGC & Serine \\
$2+2 u$ & $(2,2,0)$ & AGA & Glutamate \\
& & &
\end{tabular}




\begin{tabular}{|c|c|c|c|}
\hline $2+u+u^{2}$ & $(2,1,1)$ & $\mathrm{ATT}$ & Isoleucine \\
\hline $2+u+2 u^{2}$ & $(2,1,2)$ & GAT & Aspartate \\
\hline $2+u+3 u^{2}$ & $(2,1,3)$ & $\mathrm{CGC}$ & Arginine \\
\hline $2+2 u+u^{2}$ & $(2,2,1)$ & $\mathrm{CAG}$ & Glutamine \\
\hline $2+2 u+2 u^{2}$ & $(2,2,2)$ & $\mathrm{CAC}$ & Histidine \\
\hline $2+2 u+3 u^{2}$ & $(2,2,3)$ & GAC & Aspartate \\
\hline $2+3 u$ & $(2,3,0)$ & GTT & Valine \\
\hline $2+3 u+u^{2}$ & $(2,3,1)$ & ATC & Isoleucine \\
\hline $2+3 u+2 u^{2}$ & $(2,3,2)$ & GGC & Glycine \\
\hline $2+3 u+3 u^{2}$ & $(2,3,3)$ & GCT & Valine \\
\hline 3 & $(3,0,0)$ & GGA & Glycine \\
\hline $3 u$ & $(0,3,0)$ & CTA & Leucine \\
\hline $3+u$ & $(3,1,0)$ & ATG & Methionine \\
\hline $3+u+u^{2}$ & $(3,1,1)$ & $\mathrm{ACG}$ & Threonine \\
\hline $3+u+2 u^{2}$ & $(3,1,2)$ & $\mathrm{ACC}$ & Threonine \\
\hline $3+u+3 u^{2}$ & $(3,1,3)$ & AAT & Asparagine \\
\hline $3+2 u$ & $(3,2,0)$ & TCA & Serine \\
\hline $3+2 u+u^{2}$ & $(3,2,1)$ & $\mathrm{AAG}$ & Lysine \\
\hline $3+2 u+2 u^{2}$ & $(3,2,2)$ & $\mathrm{CCG}$ & Alanine \\
\hline $3+2 u+3 u^{2}$ & $(3,2,3)$ & TTG & Leucine \\
\hline $3+3 u$ & $(3,3,0)$ & CGT & Arginine \\
\hline $3+3 u+u^{2}$ & $(3,3,1)$ & $\mathrm{CTT}$ & Leucine \\
\hline $3+3 u+2 u^{2}$ & $(3,3,2)$ & $\mathrm{ACT}$ & Threonine \\
\hline $3+3 u+3 u^{2}$ & $(3,3,3)$ & $\mathrm{CCC}$ & Proline \\
\hline $1+u^{2}$ & $(1,0,1)$ & $\mathrm{AAC}$ & Asparagine \\
\hline $1+2 u^{2}$ & $(1,0,2)$ & AGT & Serine \\
\hline $1+3 u^{2}$ & $(1,0,3)$ & TTC & Phenylalanine \\
\hline $2+u^{2}$ & $(2,0,1)$ & TAT & Tyrosine \\
\hline $2+2 u^{2}$ & $(2,0,2)$ & TGT & Cysteine \\
\hline $2+3 u^{2}$ & $(2,0,3)$ & GTA & Valine \\
\hline $3+u^{2}$ & $(3,0,1)$ & TCC & Serine \\
\hline $3+2 u^{2}$ & $(3,0,2)$ & GGT & Glycine \\
\hline $3+3 u^{2}$ & $(3,0,3)$ & $\mathrm{AAA}$ & Lysine \\
\hline$u^{2}$ & $(0,0,1)$ & CTG & Leucine \\
\hline $2 u^{2}$ & $(0,0,2)$ & TCT & Leucine \\
\hline $3 u^{2}$ & $(0,0,3)$ & GTC & Valine \\
\hline
\end{tabular}

Definition 2.1. Let $a, b \in \mathbb{Z}_{4}$, then we define a distance called Gray distance by $d_{G}(a, b)=$ $d_{H}(\phi(a), \phi(b))$, where $\phi(a), \phi(b) \in D^{3}$. Note that it can be extended upto length $n$. Hence the map $\phi$ is a distance preserving map from $\left(R^{n}, d_{G}\right)$ to $\left(D^{3 n}, d_{H}\right)$.

Example 2.2. Let $a=1+u+u^{2}$ and $b=2+2 u+2 u^{2}$, then

$$
d_{G}(a, b)=d_{H}(G G G, C A C)=3 .
$$

Definition 2.3. If $\mathcal{C}$ is invariant under the cyclic shift operator $\delta: R^{n} \rightarrow R^{n}$ given by $\delta\left(c_{1}, c_{2}, \cdots, c_{n}\right)=$ $\left(c_{n}, c_{1}, \cdots, c_{n-1}\right)$, then the code $\mathcal{C}$ is called a cyclic code of length $n$.

Definition 2.4. Let $\mathcal{C}$ be a code of arbitrary length $n$ over any finite set $\mathcal{A}$. Then $\mathcal{C}$ is called reversible code if it remains invariant under the reversal of each codeword, i.e., if $c=\left(c_{1}, c_{2}, \ldots, c_{n}\right) \in \mathcal{C}$, then $c^{R}=\left(c_{n}, c_{n-1}, \ldots, c_{1}\right) \in \mathcal{C}$.

While working with cyclic and reversible codes we need to deal with a polynomial called selfreciprocal polynomial defined in the following definition.

Definition 2.5. Let $p(x)=a_{0}+a_{1} x+\cdots+a_{n} x^{n}$ be a polynomial of degree $n$. The polynomial $g(x)=$ $a_{n}+a_{n-1} x+\cdots+a_{0} x^{n}$ is called reciprocal polynomial of $p(x)$. A polynomial $p(x)$ over $R$ is said to be self-reciprocal polynomial if $p(x)=p^{*}(x)$, where $p^{*}(x)=x^{n} p\left(\frac{1}{x}\right)$. 
We follow the definition of a DNA code from [12]. A code $C$ is called a DNA code if it satisfies some of the following properties:

(i) The Hamming distance constraint is defined as $d_{H}(a, b) \geq d$ for all $a, b \in \mathcal{C}$ and $a \neq b$ for some predefined distance $d$.

(ii) The reverse constraint is defined as $d_{H}\left(a^{R}, b\right) \geq d$ for all $a, b \in \mathcal{C}$ and $a \neq b$ for some predefined distance $d$ and where $a^{R}$ denotes the reverse sequence of alphabets in $a$.

(iii) The reverse complement constraint is defined as $d_{H}\left(a^{R}, \bar{b}\right) \geq d$ for all $a, b \in \mathcal{C}, a \neq b$ for some predefined distance $d$ and where $\bar{b}$ denotes a word in which each alphabet of $b$ is replaced by its Watson-Crick complement. DNA code satisfying reverse complement constraint is called the reverse complement DNA code.

(iv) The fixed GC-content constraint specifies that each codeword must have fixed number of $G$ 's and $C$ 's. Generally this fixed number is $\left\lfloor\frac{n}{2}\right\rfloor$ where $n$ denotes the length of codewords.

Definition 2.6. Let $\mathcal{C}$ be a code of arbitrary length $n$ over $D$. Then $\mathcal{C}$ is called reverse complement code if it contains both the reverse and the complement of every codeword in $\mathcal{C}$, that is, if $\left(c_{1}, c_{2}, \ldots, c_{n}\right) \in$ $\mathcal{C}$, then $\left(\overline{c_{n}}, \overline{c_{n-1}}, \ldots, \overline{c_{1}}\right) \in \mathcal{C}$.

It is interesting to observe that one-to-one correspondence we define is a customized gray map between the DNA codons and elements of the ring $R$. The elements of ideal $A_{2}$ correspond only to self reversible DNA codons. This map take care of GC content for the ideals $A_{2 u^{2}}$ and $A_{2 u}$. The GC content is 30 to 50 percentage in these ideals. Further, the map satisfies the following property.

Lemma 2.7. Let $a \in R$, then $\bar{a}=a+2\left(1+u+u^{2}\right)$.

\section{Cyclic DNA codes over $R$}

In this section, we study the reverse and the reverse complement cyclic codes over $R$. For this, we will use the following Lemmas.

Lemma 3.1. [1] Let $p(x)$ and $q(x)$ be polynomials over $\mathbb{Z}_{4}$ with $\operatorname{deg} p(x) \geq \operatorname{deg} q(x)$. Then

(i) $[p(x) q(x)]^{*}=p^{*}(x) q^{*}(x)$

(ii) $[p(x)+q(x)]^{*}=p^{*}(x)+x^{(\operatorname{deg} p(x)-\operatorname{deg} q(x))} q^{*}(x)$.

Lemma 3.2. [3] Let $\mathcal{C}=\langle p(x)\rangle$ be a cyclic code of odd length $n$ over $\mathbb{Z}_{4}$; where $p(x)$ is a monic polynomial of degree $r$ over $\mathbb{Z}_{4}$. Then $\mathcal{C}$ is reversible if and only if $p(x)$ is self reciprocal polynomial.

Now, we are able to prove the following result which will be useful thereafter.

Lemma 3.3. Let $p_{1}(x), p_{2}(x), \ldots, p_{r}(x)$ be polynomials over $\mathbb{Z}_{4}$ with $\operatorname{deg} p_{i}(x)=k_{i}$ and $k_{r} \leq k_{r-1} \leq$ $\cdots \leq k_{1}$, then

$$
\begin{aligned}
& \text { (i) }\left[p_{1}(x) p_{2}(x) \cdots p_{r}(x)\right]^{*}=p_{1}^{*}(x) p_{2}^{*}(x) \cdots p_{r}^{*}(x) \text { and } \\
& \text { (ii) }\left[p_{1}(x)+p_{2}(x)+\cdots+p_{r}(x)\right]^{*}=p_{1}^{*}(x)+x^{k_{1}-k_{2}} p_{2}^{*}(x)+\cdots+x^{k_{1}-k_{i}} p_{i}^{*}(x)+\cdots+x^{k_{1}-k_{r}} p_{r}^{*}(x) \text {. }
\end{aligned}
$$

Proof. We prove this by induction on $r$. By Lemma 3.1, this is true for $r=2$. Let us assume that this is true for less than or equal to $l$, that is,

$$
\begin{aligned}
{\left[p_{1}(x) p_{2}(x) \cdots p_{l}(x)\right]^{*} } & =p_{1}^{*}(x) p_{2}^{*}(x) \cdots p_{l}^{*}(x), \\
{\left[p_{1}(x)+p_{2}(x)+\cdots+p_{l}(x)\right]^{*} } & =p_{1}^{*}(x)+x^{k_{1}-k_{2}} p_{2}^{*}(x)+\cdots+x^{k_{1}-k_{l}} p_{l}^{*}(x) .
\end{aligned}
$$


Now, we will check whether the hypothesis is true for $r=l+1$. By using hypothesis for $r=2$ and $r=l$ consecutively,

$$
\begin{aligned}
{\left[p_{1}(x) p_{2}(x) \cdots p_{l}(x) p_{l+1}(x)\right]^{*} } & =\left[p_{1}(x) p_{2}(x) \cdots p_{l}(x)\right]^{*} p_{l+1}^{*}(x) \\
& =p_{1}^{*}(x) p_{2}^{*}(x) \cdots p_{l+1}^{*}(x)
\end{aligned}
$$

Repeat the same process to prove the second identity.

$$
\begin{aligned}
{\left[p_{1}(x)+p_{2}(x)+\cdots+p_{l+1}(x)\right]^{*} } & =\left[p_{1}(x)+p_{2}(x)+\cdots+p_{l}(x)\right]^{*}+x^{\left(k_{1}-k_{l+1}\right)} p_{l+1}^{*}(x) \\
& =p_{1}^{*}(x)+x^{k_{1}-k_{2}} p_{2}^{*}(x)+\cdots+x^{k_{1}-k_{l}} p_{l}^{*}(x)+x^{\left(k_{1}-k_{l+1}\right)} p_{l+1}^{*}(x)
\end{aligned}
$$

Hence the induction hypothesis is true for $r=l+1$. Therefore, by mathematical induction, the statement is true for all positive integer $r$.

Proposition 3.4. Let $p_{i}(x)$ and $q_{i}(x)$ be in $\mathbb{Z}_{4}[x]$ for $i=1,2$ and 3 . If the following equality holds

$$
p_{1}(x)+u p_{2}(x)+u^{2} p_{3}(x)=q_{1}(x)+u q_{2}(x)+u^{2} q_{3}(x),
$$

then $p_{i}(x)=q_{i}(x)$ for $i=1,2$ and 3 .

Proof. Assume that equation (1) is true. Multiplying it by $u^{2}$ and using $u^{3}=0$, we have $u^{2} p_{1}(x)=$ $u^{2} q_{1}(x)$. Therefore $p_{1}(x)=q_{1}(x)$, because $p_{1}(x), q_{1}(x) \in \mathbb{Z}_{4}[x]$.

Again multiplying (1) by $u$ and substituting $p_{1}(x)=q_{1}(x)$, we get $u^{2} p_{2}(x)=u^{2} q_{2}(x)$ and hence $p_{2}(x)=q_{2}(x)$. Similarly, we get $p_{3}(x)=q_{3}(x)$.

Now, we study the reverse constraint of one generator cyclic code of odd length $n$ over $R$. For this, first we need the following result which states one generator cyclic codes of odd length $n$ over the ring $R$. This result with its proof can be found in [15].

Theorem 3.5. [15] Let $\mathcal{C}$ be a cyclic code of odd length $n$ over $R$. If $\mathcal{C}=\left\langle h_{1}(x)+u g_{1}(x)+\right.$ $\left.u^{2} b_{1}(x), u h_{2}(x)+u^{2} b_{2}(x), u^{2} h_{3}(x)\right\rangle$ and $h_{1}(x)$ and $h_{3}(x)$ are equal, then

$$
\mathcal{C}=\left\langle h_{1}(x)+u g_{1}(x)+u^{2} b_{1}(x)\right\rangle .
$$

Definition 3.6. Let $R$ be a ring and $p(x) \in R[x]$ is called regular polynomial if it is not a zero divisor in $R[x]$.

Theorem 3.7. Let $\mathcal{C}$ be a cyclic code of odd length $n$ over the ring $R$. Let $p(x)$ be a regular polynomial in $\mathbb{Z}_{4}[x]$ and $\mathcal{C}=\left\langle p(x)+u q(x)+u^{2} h(x)\right\rangle$ where $p(x), q(x)$ and $h(x)$ are in $\mathbb{Z}_{4}[x]$. If $\operatorname{deg} p(x)=r$, $\operatorname{deg} q(x)=s$ and $\operatorname{deg} h(x)=t$ with $r \geq s \geq t$, then $\mathcal{C}$ is reversible if and only if

(a) $p(x)$ is a self reciprocal polynomial.

(b) (i) $x^{r-s} q^{*}(x)=q(x)$ and $x^{r-t} h^{*}(x)=h(x)$, or

(ii) $x^{r-s} q^{*}(x)=p(x)+q(x)$ and $x^{r-t} h^{*}(x)=q(x)+h(x)$, or

(iii) $x^{r-s} q^{*}(x)=p(x)+q(x)$ and $x^{r-t} h^{*}(x)=p(x)+q(x)+h(x)$, or

(iv) $x^{r-s} q^{*}(x)=q(x)$ and $x^{r-t} h^{*}(x)=p(x)+h(x)$.

Proof. Let us assume that $\mathcal{C}$ is a reversible code over $R$. Then $\mathcal{C} \bmod u=\langle p(x)\rangle$ is a reversible code over $\mathbb{Z}_{4}$. Therefore, by Lemma 3.2 we have that $p(x)$ is a self reciprocal polynomial in $\mathbb{Z}_{4}[x]$.

Now, by Lemma 3.3 we have

$$
\begin{aligned}
{\left[p(x)+u q(x)+u^{2} h(x)\right]^{*} } & =p^{*}(x)+u x^{r-s} q^{*}(x)+u^{2} x^{r-t} h^{*}(x) \\
& =p(x)+u x^{r-s} q^{*}(x)+u^{2} x^{r-t} h^{*}(x) \\
& =\left(p(x)+u q(x)+u^{2} h(x)\right) k(x) \in \mathcal{C} .
\end{aligned}
$$


Since the degrees of both sides are the same, we have $k(x)$ is a constant in $R$, i.e., $k=k_{1}+u k_{2}+u^{2} k_{3}$ and hence

$$
\begin{aligned}
p(x)+u x^{r-s} q^{*}(x)+u^{2} x^{r-t} h^{*}(x) & =\left(p(x)+u q(x)+u^{2} h(x)\right) k \\
& =\left(p(x)+u q(x)+u^{2} h(x)\right)\left(k_{1}+u k_{2}+u^{2} k_{3}\right) \\
& =p(x) k_{1}+u\left(p(x) k_{2}+q(x) k_{1}\right)+u^{2}\left(p(x) k_{3}+q(x) k_{2}+h(x) k_{1}\right)
\end{aligned}
$$

Now by Proposition 3.4, we get $k_{1}=1$ and each $k_{2}$ and $k_{3}$ have 4 possibilities. Therefore, we have 16 possible cases, i.e., conditions for $k=1,1+u, 1+2 u, 1+3 u, 1+u+u^{2}, 1+u+2 u^{2}, 1+u+3 u^{2}, 1+$ $2 u+u^{2}, 1+2 u+2 u^{2}, 1+2 u+3 u^{2}, 1+3 u+u^{2}, 1+3 u+2 u^{2}, 1+3 u+3 u^{2}, 1+u^{2}, 1+2 u^{2}, 1+3 u^{2}$. We have investigated all these cases by substituting values of $k$ in (2). Then we get the following summarized results:

\begin{tabular}{|c|c|}
\hline Constant $k$ & Conditions Obtained \\
\hline$k=1$ & $x^{r-s} q^{*}(x)=q(x)$ and $x^{r-t} h^{*}(x)=h(x)$ \\
\hline$k=1+u$ & $x^{r-s} q^{*}(x)=p(x)+q(x)$ and $x^{r-t} h^{*}(x)=q(x)+h(x)$ \\
\hline$k=1+2 u$ & $2 x^{r-s} q^{*}(x)=2 q(x)$ and $2 x^{r-t} h^{*}(x)=2 h(x)$ \\
\hline$k=1+3 u$ & $2 x^{r-s} q^{*}(x)=2 p(x)+2 q(x)$ and $2 x^{r-t} h^{*}(x)=2 q(x)+2 h(x)$ \\
\hline$k=1+u+u^{2}$ & $x^{r-s} q^{*}(x)=p(x)+q(x)$ and $x^{r-t} h^{*}(x)=p(x)+q(x)+h(x)$ \\
\hline$k=1+u+2 u^{2}$ & $2 x^{r-s} q^{*}(x)=2 p(x)+2 q(x)$ and $2 x^{r-t} h^{*}(x)=2 q(x)+2 h(x)$ \\
\hline$k=1+u+3 u^{2}$ & $x^{r-s} q^{*}(x)=p(x)+q(x)$ and $2 x^{r-t} h^{*}(x)=2 p(x)+2 q(x)+2 h(x)$ \\
\hline$k=1+2 u+u^{2}$ & $2 x^{r-s} q^{*}(x)=2 q(x)$ and $2 x^{r-t} h^{*}(x)=2 p(x)+2 h(x)$ \\
\hline$k=1+2 u+2 u^{2}$ & $2 x^{r-s} q^{*}(x)=2 q(x)$ and $2 x^{r-t} h^{*}(x)=2 h(x)$ \\
\hline$k=1+2 u+3 u^{2}$ & $2 x^{r-s} q^{*}(x)=2 q(x)$ and $2 x^{r-t} h^{*}(x)=2 p(x)+2 h(x)$ \\
\hline$k=1+3 u+u^{2}$ & $2 x^{r-s} q^{*}(x)=2 p(x)+2 q(x)$ and $2 x^{r-t} h^{*}(x)=2 p(x)+2 q(x)+2 h(x)$ \\
\hline$k=1+3 u+2 u^{2}$ & $2 x^{r-s} q^{*}(x)=2 p(x)+2 q(x)$ and $2 x^{r-t} h^{*}(x)=2 q(x)+2 h(x)$ \\
\hline$k=1+3 u+3 u^{2}$ & $2 x^{r-s} q^{*}(x)=2 p(x)+2 q(x)$ and $2 x^{r-t} h^{*}(x)=2 p(x)+2 q(x)+2 h(x)$ \\
\hline$k=1+u^{2}$ & $x^{r-s} q^{*}(x)=q(x)$ and $x^{r-t} h^{*}(x)=p(x)+h(x)$ \\
\hline$k=1+2 u^{2}$ & $x^{r-s} q^{*}(x)=q(x)$ and $2 x^{r-t} h^{*}(x)=2 h(x)$ \\
\hline$k=1+3 u^{2}$ & $x^{r-s} q^{*}(x)=q(x)$ and $2 x^{r-t} h^{*}(x)=2 p(x)+2 h(x)$ \\
\hline
\end{tabular}

From the above table, we can observe that there are 12 similar cases. Therefore, we can reduce above conditions into following four cases.

(i) $x^{r-s} q^{*}(x)=q(x)$ and $x^{r-t} h^{*}(x)=h(x)$, or

(ii) $x^{r-s} q^{*}(x)=p(x)+q(x)$ and $x^{r-t} h^{*}(x)=q(x)+h(x)$, or

(iii) $x^{r-s} q^{*}(x)=p(x)+q(x)$ and $x^{r-t} h^{*}(x)=p(x)+q(x)+h(x)$, or

(iv) $x^{r-s} q^{*}(x)=q(x)$ and $x^{r-t} h^{*}(x)=p(x)+h(x)$.

For the converse part, let us assume that the hypothesis is true. Since $\mathcal{C}$ is a cyclic code over $R$, it is enough to show that $\left[p(x)+u q(x)+u^{2} h(x)\right]^{*}$ belong to $\mathcal{C}$.

$$
\begin{aligned}
{\left[p(x)+u q(x)+u^{2} h(x)\right]^{*} } & =p^{*}(x)+u x^{r-s} q^{*}(x)+u^{2} x^{r-t} h^{*}(x) \\
& =p(x)+u x^{r-s} q^{*}(x)+u^{2} x^{r-t} h^{*}(x) .
\end{aligned}
$$

Now using conditions in the hypothesis, we have

$$
\left[p(x)+u q(x)+u^{2} h(x)\right]^{*}=\left(p(x)+u q(x)+u^{2} h(x)\right) k \in \mathcal{C}
$$

where the constant $k \in\left\{1,1+u, 1+2 u, 1+3 u, 1+u+u^{2}, 1+u+2 u^{2}, 1+u+3 u^{2}, 1+2 u+u^{2}, 1+2 u+\right.$ $\left.2 u^{2}, 1+2 u+3 u^{2}, 1+3 u+u^{2}, 1+3 u+2 u^{2}, 1+3 u+3 u^{2}, 1+u^{2}, 1+2 u^{2}, 1+3 u^{2}\right\} \subseteq R$. Therefore, $\mathcal{C}$ is a reversible cyclic code in $R[x]$. 
By using Table 1 and Lemma 2.7, we have the following result.

Theorem 3.8. Let $\mathcal{C}=\left\langle p(x)+u q(x)+u^{2} h(x)\right\rangle$ be a cyclic code over $R$ where $p(x), q(x), h(x) \in \mathbb{Z}_{4}[x]$. If $\operatorname{deg} p(x)=r, \operatorname{deg} q(x)=s$ and $\operatorname{deg} h(x)=t$ with $r \geq s \geq t$, then $\mathcal{C}$ is a reverse complement code if and only if

1. the element $\frac{2\left(1+u+u^{2}\right)\left(x^{n}-1\right)}{(x-1)} \in \mathcal{C}$ and

2. the cyclic code $\mathcal{C}$ is reversible.

Proof. Let $\mathcal{C}$ be the code satisfying the hypothesis. Let $c(x)=c_{0}+c_{1} x+\cdots+c_{k} x^{k} \in \mathcal{C}$. Since $\mathcal{C}$ is reversible, $c^{*}(x)=c_{k}+c_{k-1} x+\cdots+c_{0} x^{k} \in \mathcal{C}$. As $\mathcal{C}$ is an ideal of $\frac{R[x]}{\left\langle x^{n}-1\right\rangle}$, so $x^{n-k-1} c^{*}(x)=$ $c_{k} x^{n-k-1}+c_{k-1} x^{n-k}+\cdots+c_{0} x^{n-1}$ belongs to $\mathcal{C}$. By hypothesis $2\left(1+u+u^{2}\right) \frac{\left(x^{n}-1\right)}{(x-1)} \in \mathcal{C}$, therefore $2\left(1+u+u^{2}\right) \frac{\left(x^{n}-1\right)}{(x-1)}+x^{n-k-1} c^{*}(x) \in \mathcal{C}$. Then

$$
\begin{aligned}
2\left(1+u+u^{2}\right) \frac{\left(x^{n}-1\right)}{(x-1)}+x^{n-k-1} c^{*}(x)= & 2\left(1+u+u^{2}\right)\left(1+x+x^{2}+\cdots+x^{n-k-2}\right) \\
& +\left(c_{k}+2\left(1+u+u^{2}\right)\right) x^{n-k-1}+\cdots \\
& +\left(c_{0}+2\left(1+u+u^{2}\right)\right) x^{n-1} \\
= & 2\left(1+u+u^{2}\right)\left(1+x+x^{2}+\cdots+x^{n-k-2}\right) \\
& +\overline{c_{k}} x^{n-k-1}+\overline{c_{k-1}} x^{n-k}+\cdots+\overline{c_{0}} x^{n-1} \\
2\left(1+u+u^{2}\right) \frac{\left(x^{n}-1\right)}{(x-1)}+x^{n-k-1} c^{*}(x)= & \overline{c^{*}(x)}
\end{aligned}
$$

Hence, we conclude that $\mathcal{C}$ is a reverse complement code.

Conversely, we assume that $\mathcal{C}$ is a reverse complement code, i.e., if $c(x) \in \mathcal{C}$, then $\overline{c^{*}(x)} \in \mathcal{C}$. First we observe that since $\mathcal{C}$ is linear this implies that the element $a(x)=0 \in \mathcal{C}$ and therefore

$$
\overline{a(x)}=2\left(1+u+u^{2}\right)\left(1+x+\cdots+x^{n-1}\right)=2\left(1+u+u^{2}\right) \frac{\left(x^{n}-1\right)}{(x-1)} \in \mathcal{C} .
$$

Now, let $c(x)=c_{0}+c_{1} x+\cdots+c_{k} x^{k} \in \mathcal{C}$, then

$$
\begin{aligned}
\overline{c^{*}(x)}= & 2\left(1+u+u^{2}\right)\left(1+x+\cdots+x^{n-k-2}\right)+x^{n-k-1} \overline{c_{k}}+\cdots+\overline{c_{0}} \\
= & 2\left(1+u+u^{2}\right)\left(1+x+\cdots+x^{n-k-2}\right)+x^{n-k-1}\left(c_{k}+2\left(1+u+u^{2}\right)\right)+ \\
& \cdots+\left(c_{0}+2\left(1+u+u^{2}\right)\right) x^{n-1}
\end{aligned}
$$

Adding $2\left(1+u+u^{2}\right) \frac{\left(x^{n}-1\right)}{(x-1)}$ to the above equation, we get

$$
\begin{aligned}
\overline{c^{*}(x)}+2\left(1+u+u^{2}\right) \frac{\left(x^{n}-1\right)}{(x-1)} & =c_{k} x^{n-k-1}+c_{k-1} x^{n-k}+\cdots+c_{0} x^{n-1} \\
& =x^{n-k-1}\left(c_{k}+c_{k-1} x+\cdots+c_{0} x^{k}\right)
\end{aligned}
$$

Multiplying both side by $x^{k+1}$, we get

$$
x^{k+1}\left(\overline{c^{*}(x)}+2\left(1+u+u^{2}\right) \frac{\left(x^{n}-1\right)}{(x-1)}\right)=c_{k}+c_{k-1} x+\cdots+c_{0} x^{k}=c^{*}(x) .
$$

Thus, we have $\mathcal{C}$ is reversible code. 
Theorem 3.9. Let $\mathcal{C}$ be a cyclic code of odd length $n$ which satisfies the conditions of Theorem 3.8, then $\mathcal{C}$ is a DNA code.

Proof. Combining the proof of Theorem 3.7 and Theorem 3.8, we get the result.

Example 3.10. Let $x=\left(2 u+2 u^{2}, 2+2 u^{2}, 2+2 u\right)$ and $y=\left(2+2 u, 2+2 u^{2}, 2 u+2 u^{2}\right)$. We define a code $\mathcal{C}$ consisting of all cyclic shifts and linear combinations of the vectors $x$ and $y$ over $R$. Thus we obtain corresponding DNA code of parameters $(9,32,4)$ as follows,

$\begin{array}{llll}C T C T G T A G A & \text { GAGCACGAG } & \text { CACGAGGAG } & \text { CTCCTCGTG } \\ \text { GTGGTGGTG } & \text { AGATGTCTC } & \text { TCTACAGAG } & \text { CACCACCAC } \\ \text { GTGCTCCTC } & \text { GAGGAGCAC } & \text { CTCGTGCTC } & \text { GAGACATCT } \\ \text { ACAGAGTCT } & \text { CTCAGATGT } & \text { AGAGTGAGA } & \text { CACTCTTCT } \\ \text { TCTTCTCAC } & \text { TGTGTGTGT } & \text { TCTGAGACA } & \text { GTGTGTTGT } \\ \text { TGTAGACTC } & \text { ACAACACAC } & \text { TGTTGTGAG } & \text { ACATCTGAG } \\ \text { CACACAACA } & \text { AGACTCTGT } & \text { ACACACACA } & \text { AGAAGAGTG } \\ \text { GTGAGAAGA } & \text { TCTCACTCT } & \text { GAGTCTACA } & \text { TGTCTCAGA }\end{array}$

Example 3.11. Let $x=\left(2,2+2 u, 2 u, 2+2 u+2 u^{2}\right)$, then we define a code generated by a generator matrix consisting of the cyclic shifts of the vector $x$ over $R$. Thus, we have a DNA code of parameters $(12,16,6)$ as follows,

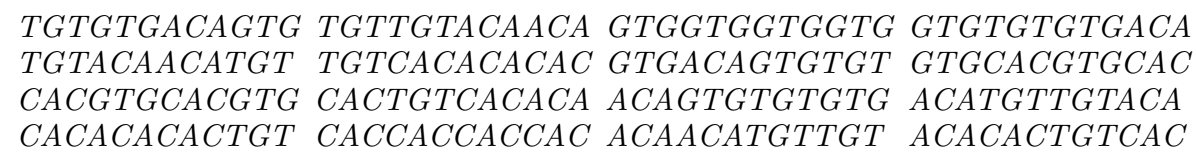

\section{Binary image of elements in $R$}

In this section, we define binary images of elements of the ring $R$ which will be useful for DNA computing. An element in the ring $R$ is of the form $a+u b+u^{2} c$; where $a, b, c \in \mathbb{Z}_{4}$. Now, we can define a map between $R$ and $\mathbb{Z}_{2}$. A one-to-one correspondence between $R$ and $\mathbb{Z}_{4}^{3}$ is defined as $\psi: R \rightarrow \mathbb{Z}_{4}^{3}$ with $\left(a+u b+u^{2} c\right) \mapsto(a, b, c)$ where $a, b, c \in \mathbb{Z}_{4}$.

We define a Gray map $\varphi: \mathbb{Z}_{4} \rightarrow \mathbb{Z}_{2}^{2}$ using 2-adic expansion of elements in $\mathbb{Z}_{4}$ which are as follows:

\begin{tabular}{cccc}
$\mathrm{c}$ & $\pi(c)$ & $\rho(c)$ & $v(c)$ \\
\hline 0 & 0 & 0 & 0 \\
1 & 1 & 0 & 1 \\
2 & 0 & 1 & 1 \\
3 & 1 & 1 & 0
\end{tabular}

We have $\varphi(c)=(\rho(c), v(c))$ for all $c \in \mathbb{Z}_{4}$ (as in [13]). Therefore,

$$
0 \rightarrow 00,1 \rightarrow 01,2 \rightarrow 11,3 \rightarrow 10
$$

For any $v \in \mathbb{Z}_{4}$, the Lee weight $w_{L}(v)$ is defined as $\min (v, 4-v)$. This Lee weight can be extended to the ring $R$ as follow: for $x=a+u b+u^{2} c$ Lee weight of $x$ is defined as $w_{L}(x)=w_{L}(a, b, c)$. The Hamming distance $d_{H}\left(c_{1}, c_{2}\right)$ between two codewords $c_{1}$ and $c_{2}$ is the Hamming weight of the codeword $w_{H}\left(c_{1}-c_{2}\right)$ that is the number of non zero element in $\left(c_{1}-c_{2}\right)$. Define $\chi: R \rightarrow \mathbb{Z}_{2}^{6}$ by $\chi\left(a+u b+u^{2} c\right)=$ $(\rho(a), v(a), \rho(b), v(b), \rho(c), v(c))$. The map $\chi$ is clearly a linear map.

Lemma 4.1. The Gray map from $R^{n}$ to $\mathbb{Z}_{2}^{6 n}$ is a distance preserving map. 
Proof. Let $x_{1}, x_{2}$ be two elements of $R^{n}$. Then $d_{L}\left(x_{1}, x_{2}\right)=w_{L}\left(x_{1}-x_{2}\right)$. Since the map is bijective, we have $w_{L}\left(x_{1}-x_{2}\right)=w_{H}\left(\chi\left(x_{1}-x_{2}\right)\right)=w_{H}\left(\chi\left(x_{1}\right)-\chi\left(x_{2}\right)\right)$. Therefore, the Gray map $\chi$ is distance preserving.

Given an element $c$ of length $n$ in a cyclic code $\mathcal{C}$. If the cyclic shift $\delta(c) \in \mathcal{C}$ then $\psi(\delta(\mathcal{C}))$ will be quasi cyclic code of length $3 n$ with index 3 over $\mathbb{Z}_{4}^{3 n}$. Then it can be easily seen that $\varphi(\psi(\delta(c)))$ satisfies quasi cyclic shift of length $6 n$ with index 6 over $\mathbb{Z}_{2}^{6 n}$. Thus we have the following theorem.

Theorem 4.2. If $\mathcal{C}$ is a cyclic code of length $n$, then its image is a quasi cyclic code of length $6 n$ and with index 6 over $\mathbb{Z}_{2}$.

\section{Skew cyclic codes over $R$}

Let $\theta$ be a non-trivial automorphism defined by $\theta: R \rightarrow R$ such that $\left(a+u b+u^{2} c\right) \mapsto a-u b+u^{2} c$. The order of $\theta$ is 2 that is $\theta\left(\theta\left(a+u b+u^{2} c\right)\right)=a+u b+u^{2} c$. The ring $R[x, \theta]=\left\{a_{0}+a_{1} x+a_{2} x^{2}+\cdots+\right.$ $\left.a_{n-1} x^{n-1}: a_{i} \in R, n \in \mathbb{N}\right\}$, a non commutative ring with usual addition and multiplication defined as $a x^{i} \cdot b x^{j}=a \theta^{i}(b) x^{i+j}$ is called skew polynomial ring.

Definition 5.1. A set $\mathcal{C}$ of codewords over $R^{n}$ is skew cyclic code if it satisfies the following

(i) $\mathcal{C}$ is a submodule over $R$.

(ii) Whenever $\left(c_{0}, c_{1}, \ldots, c_{n-1}\right) \in \mathcal{C}$ then $\left(\theta\left(c_{n-1}\right), \theta\left(c_{0}\right), \ldots, \theta\left(c_{n-2}\right)\right) \in \mathcal{C}$.

Let $p(x)+\left\langle x^{n}-1\right\rangle \in R_{\theta}=R[x, \theta] /\left\langle x^{n}-1\right\rangle$ and $r(x) \in R[x, \theta]$, then define multiplication as $r(x)\left(p(x)+\left\langle x^{n}-1\right\rangle\right)=r(x) p(x)+\left\langle x^{n}-1\right\rangle$, for any $r(x) \in R[x, \theta]$. Clearly $R_{\theta}$ is left $R[x, \theta]-$ module.

Theorem 5.2. A code $\mathcal{C}$ over $R$ is a skew cyclic code of length $n$ if and only if $\mathcal{C}$ is left ideal of $R[x, \theta]$ module $R_{\theta}^{n}$.

Proof. The proof is same as the proof of [7, Theorem 1].

Theorem 5.3. Let $\mathcal{C}$ be a skew cyclic code of length $n$ over $R$. If $\mathcal{C}$ contains a monic polynomial of minimal degree $p(x)$, then $\mathcal{C}=\langle p(x)\rangle$, where $p(x)$ is a right divisor of $x^{n}-1$.

Proof. The proof is same as the proof of [7, Lemma 1]].

Now, we will introduce reverse complement skew cyclic codes over $R$. For this we have to notice that the multiplication over $R[x, \theta]$ is not commutative therefore we need to see things differently. Let $c=\left(c_{0}, c_{1}, \ldots, c_{n-1}\right)$ be in $R[x, \theta]$ then reversal of $c$ denoted by $c^{R}$ is given by $c^{R}=\left(c_{n-1}, c_{n-2}, \cdots, c_{0}\right)$.

$$
\begin{aligned}
c\left(x^{-1}\right) \cdot x^{n-1} & =\left(c_{0}+c_{1} x^{-1}+\cdots+c_{n-1} x^{-n+1}\right) \cdot x^{n-1} \\
& =c_{0} \cdot x^{n-1}+c_{1} x^{-1} \cdot x^{n-1}+\cdots+c_{n-1} x^{-n+1} \cdot x^{n-1} \\
& =c_{0} \theta^{0}(1) x^{n-1}+c_{1} \theta^{-1} x^{n-1-1}+\cdots+c_{n-1} \theta^{-n+1} x^{-n+1+n-1} \\
& =c_{0} x^{n-1}+c_{1} x^{n-2}+\cdots+c_{n-1}=c^{R}
\end{aligned}
$$

As $\theta$ is an automorphism we have $\theta(1)=1$ and $\theta^{r}(1)=1$ for all $r \in \mathbb{Z}$. Note that the reciprocal polynomial $c^{*}(x)$ and $c^{R}(x)$ are different due to the operations on them. Also see $(p(x) \cdot q(x))^{R} \neq p^{R}(x) \cdot q^{R}(x)$ as in Lemma 3.3. If $p^{R}(x)$ coincide with $\left(r p^{R}(x)\right)$, then $p(x)$ is called self reciprocal polynomial where $r$ is a constant in $R$.

Theorem 5.4. Let $\mathcal{C}=\langle p(x)\rangle$ be a skew cyclic code of length $n$, where $p(x)$ is a monic polynomial. Then if $\mathcal{C}$ is reverse complement cyclic code then $p(x)$ is a self reciprocal polynomial and $2\left(1+u+u^{2}\right)\left(x^{n}-\right.$ 1) $/(x-1) \in \mathcal{C}$. 
Proof. Let $\mathcal{C}=\langle p(x)\rangle$ be a reverse complement cyclic code. Since $\mathcal{C}$ is linear $(0, \cdots, 0) \in \mathcal{C}$, therefore $2\left(1+u+u^{2}\right)\left(x^{n}-1\right) /(x-1) \in \mathcal{C}$. Let $p(x)=1+p_{1} x+p_{2} x^{2}+\cdots+x^{k}$. Then,

$$
\begin{aligned}
\overline{p(x)}^{R}= & \overline{\left(p\left(x^{-1}\right) \cdot x^{k-1}\right)} \\
= & 2\left(1+u+u^{2}\right)\left(1+x+\cdots+x^{n-k-2}\right)+\overline{1} x^{n-k-1}+\overline{p_{k-1}} x^{n-k}+\cdots+\overline{1} x^{n-1} \\
= & 2\left(1+u+u^{2}\right)\left(1+x+\cdots+x^{n-k-2}\right)+\left(3+2\left(1+u+u^{2}\right)\right) x^{n-k-1}+ \\
& \left(3 p_{k-1}+1+u+u^{2}\right) x^{n-k}+\cdots+\left(3+2\left(1+u+u^{2}\right)\right) x^{n-1}
\end{aligned}
$$

Now as the $\mathcal{C}$ is a reverse complement code therefore $\overline{p(x)}^{R} \in \mathcal{C}$. Using linearity of $\mathcal{C}$ we have $2\left(1+u+u^{2}\right)\left(x^{n}-1\right) /(x-1)+\overline{p(x)}^{R}=3 x^{n-k-1}+3 p_{k-1} x^{n-k}+\cdots+3 x^{n-1} \in \mathcal{C}$.

Multiplying $x^{k+1-n}$ both side and making use of $\mathcal{C}$ is a left ideal of $R[x, \theta]$-module $R_{\theta}^{n}$ we get,

$\left(2\left(1+u+u^{2}\right)\left(x^{n}-1\right) /(x-1)+\overline{p(x)}^{R}\right) \cdot x^{k+1-n}=3\left(\theta^{n-k-1}(1)+p_{k-1} \theta^{n-k}(1) x+\cdots+\theta^{n-1}(1) x^{k}\right)$ $=3\left(1+p_{k-1} x+\cdots+x^{k}\right)=3 p^{R}(x) \in \mathcal{C}$. Since $\mathcal{C}=\langle p(x)\rangle$ implies $3 p^{R}(x)=h(x) p(x)$ for some $h(x) \in R[x, \theta]$ degree of $p(x)$ and $p^{R}(x)$ is same implies $h(x)$ is constant. Hence $p(x)$ is self reciprocal.

Example 5.5. Let $p(x)=a+b x+c x^{2}+d x^{3}$, where $a=1+u+u^{2}, b=2+2 u+2 u^{2}=c$ and $d=1+3 u+u^{2}$. We define a code $\mathcal{C}$ obtained by using $G=\left[\begin{array}{c}p(x) \\ x p(x) \\ x^{2} p(x) \\ x^{3} p(x)\end{array}\right]$ as a generator matrix. Then $\mathcal{C}$ correspond to DNA code which is reverse complement skew cyclic code of length 21 and $|C|=4^{8} \cdot 2^{2}$.

\section{Examples}

Example 6.1. For $n=9, x^{9}-1=(x+3)\left(x^{2}+x+1\right)\left(x^{6}+x^{3}+1\right)$, let $\mathcal{C}=\left\langle g(x)+u p(x)+u^{2} h(x)\right\rangle$, where

\begin{tabular}{|c|c|}
\hline$C$ & $u$ \\
\hline GTAGTAGTAGTAGTAGTAGTAGTAGTA & $G G A G G A G G A G G A G G A G G A G G A G G A G G A$ \\
\hline TCATCATCATCATCATCATCATCATCA & TCGTCGTCGTCGTCGTCGTCGTCGTCG \\
\hline$\triangle A A A A A A A A A A A A A A A A A A A A A A A A A A$ & $G C A G C A G C A G C A G C A G C A G C A G C A G C A$ \\
\hline СТАCTACTACTACTACTACTACTACTA & $A C G A C G A C G A C G A C G A C G A C G A C G A C G$ \\
\hline 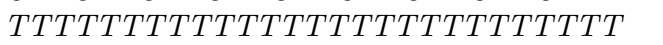 & GATGATGATGATGATGATGATGATGAT \\
\hline ATAATA ATA ATA ATAATAATAATAATA & $A G A A G A A G A A G A A G A A G A A G A A G A A G A$ \\
\hline 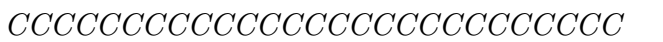 & $G A G G A G G A G G A G G A G G A G G A G G A G G A G$ \\
\hline$\triangle C A A C A A C A A C A A C A A C A A C A A C A A C A$ & AАTAATAATAATAATAATAATAATAAT \\
\hline$A A G A A G A A G A A G A A G A A G A A G A A G$ & $G A C G A C G A C G A C G A C G A C G A C G A C G A C$ \\
\hline$A A C A A C A A C A A C A A C A A C A A C A A C A A C$ & TTATTATTATTATTATTATTATTATTA \\
\hline TGATGATGATGATGATGATGATGATGA & GTTGTTGTTGTTGTTGTTGTTGTTGTT \\
\hline ТАTTATTATTATTATTATTATTATTAT & TAGTAGTAGTAGTAGTAGTAGTAGTAG \\
\hline ТАСТАСТАСТАСТАСТАСТАСТАСТАС & GTGGTGGTGGTGGTGGTGGTGGTGGTG \\
\hline TTGTTGTTGTTGTTGTTGTTGTTGTTG & ТTСТTСТТСТТСТТСТТСТТСТТСТТС \\
\hline TGTTGTTGTTGTTGTTGTTGTTGTTGT & GTCGTCGTCGTCGTCGTCGTCGTCGTC \\
\hline$T G G T G G T G G T G G T G G T G G T G G T G G T G G$ & $T G C T G C T G C T G C T G C T G C T G C T G C T G C$ \\
\hline ТСТТСТTСТTСТTСТTСТTСТTСТTCT & GGTGGTGGTGGTGGTGGTGGTGGTGGT \\
\hline ТССТССТССТССТССТССТССТССТСС & ТАATAATAATAATAATAATAATAATAA \\
\hline ATTATTATTATTATTATTATTATTATT & $G G C G G C G G C G G C G G C G G C G G C G G C G G C$ \\
\hline ATGATGATGATGATGATGATGATGATG & AGTAGTAGTAGTAGTAGTAGTAGTAGT \\
\hline$\triangle A G G A G G A G G A G G A G G A G$ & С \\
\hline
\end{tabular}
$g(x)=p(x)=h(x)=\left(x^{2}+x+1\right)\left(x^{6}+x^{3}+1\right)$. Clearly, this code satisfies $g(x)=g^{*}(x), p(x)=x^{i} p^{*}(x)$, $h(x)=x^{j} h^{*}(x)$, where $i, j=0$, gives that $\mathcal{C}$ is a reverse complement cyclic DNA code of length $n=27$ with minimum distance $d=9$ and cardinality $|\mathcal{C}|=64$. The codewords in $\mathcal{C}$ are as follows. 


\begin{abstract}
AGCAGCAGCAGCAGCAGCAGCAGCAGC $A C C A C C A C C A C C A C C A C C A C C A C C A C C$ GAAGAAGAAGAAGAAGAAGAAGAAGAA CGACGACGACGACGACGACGACGACGA CATCATCATCATCATCATCATCATCAT CACCACCACCACCACCACCACCACCAC CTGCTGCTGCTGCTGCTGCTGCTGCTG CGTCGTCGTCGTCGTCGTCGTCGTCGT

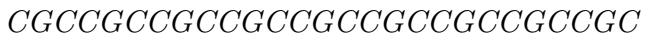
$C C G C C G C C G C C G C C G C C G C C G C C G C C G$

ACTACTACTACTACTACTACTACTACT GCCGCCGCCGCCGCCGCCGCCGCCGCC CAACAACAACAACAACAACAACAACAA CCACCACCACCACCACCACCACCACCA CAGCAGCAGCAGCAGCAGCAGCAGCAG CTTCTTCTTCTTCTTCTTCTTCTTCTT СТCСТCСТССТССТССТCСТССТССТC CGTCGTCGTCGTCGTCGTCGTCGTCGT СCTCCTCCTCCTCCTCCTCCTCCTCCT

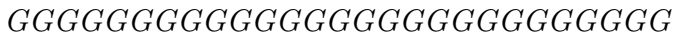

Example 6.2. For $n=5, x^{5}-1=(x+3)\left(x^{4}+x^{3}+x^{2}+x+1\right)$, let $\mathcal{C}=\left\langle g(x)+u p(x)+u^{2} h(x)\right\rangle$, where $g(x)=p(x)=h(x)=\left(x^{2}+x+1\right)\left(x^{6}+x^{3}+1\right)$. Clearly, this code satisfies $g(x)=g^{*}(x), p(x)=x^{i} p^{*}(x)$, $h(x)=x^{j} h^{*}(x)$ and hence this is a reverse complement DNA cyclic code of length $n=15$ with minimum distance $d=5$ and cardinality $|\mathcal{C}|=64$. The code $\mathcal{C}$ contains the following codewords.

\begin{tabular}{|c|c|}
\hline$T C$ & $C G$ \\
\hline GTAGTAGTAGTAGTA & $G G A G G A G G A G G A G G A$ \\
\hline TCATCATCATCATCA & $T C G T C G T C G T C G T C G$ \\
\hline$A A A A A A A A A A A A A A A$ & $G C A G C A G C A G C A G C A$ \\
\hline СТАСТАСТАСТАСТА & $A C G A C G A C G A C G A C G$ \\
\hline TTTTTTTTTTTTTTT & GATGATGATGATGAT \\
\hline ATAATAATA ATAATA & $A G A A G A A G A A G A A G A$ \\
\hline$С С С С С С С С С С С C С C$ & $G A G G A G G A G G A G G A G$ \\
\hline$A C A A C A A C A A C A A C A$ & AATAATAATA ATAAT \\
\hline$A G A A G A A G A A G A A G$ & $G A C G A C G A C G A C G A C$ \\
\hline$A A C A A C A A C A A C A A C$ & TTATTATTATTATTA \\
\hline TGATGATGATGATGA & GTTGTTGTTGTTGTT \\
\hline TATTATTATTATTAT & TAGTAGTAGTAGTAG \\
\hline ТАСТАСТАСТАСТАС & $G T G G T G G T G G T G G T G$ \\
\hline TTGTTGTTGTTGTTG & ТТСТTСТТСТТСТTC \\
\hline TGTTGTTGTTGTTGT & GTCGTCGTCGTCGTC \\
\hline$T G G T G G T G G T G G T G G$ & $T G C T G C T G C T G C T G C$ \\
\hline ТСТТСТTСТТСТТСТ & $G G T G G T G G T G G T G G T$ \\
\hline ТССТССТССТССТСС & ТААТАATA АTAATAA \\
\hline ATTATTATTATTATT & $G G C G G C G G C G G C G G C$ \\
\hline ATGATGATGATGATG & AGTAGTAGTAGTAGT \\
\hline$A G G A G G A G G A G G A G G$ & GCTGCTGCTGCTGCT \\
\hline$A G C A G C A G C A G C A G C$ & АCTACTACTACTACT \\
\hline$A C C A C C A C C A C C A C C$ & $G C C G C C G C C G C C G C C$ \\
\hline GAAGAAGAAGAAGAA & CAACAACAACAACAA \\
\hline$C G A C G A C G A C G A C G A$ & CCACCACCACCACCA \\
\hline САTCATCATCATCAT & $C A G C A G C A G C A G C A G$ \\
\hline CACCACCACCACCAC & СТTCTTCTTCTТСТT \\
\hline CTGCTGCTGCTGCTG & СТССТССТССТССТС \\
\hline CGTCGTCGTCGTCGT & CGTCGTCGTCGTCGT \\
\hline$C G C C G C C G C C G C C G C$ & ССТССТССТССТССТ \\
\hline$C C G C C G C C G C C G O$ & $G G G G G G G G$ \\
\hline
\end{tabular}

\section{Conclusion}

In this paper, we have investigated one generator cyclic codes over the ring $R=\mathbb{Z}_{4}+u \mathbb{Z}_{4}+u^{2} \mathbb{Z}_{4}$, where $u^{3}=0$ with reverse and reverse complement constraint. We have determined a mapping between codons and elements of $R$. We have studied binary image and skew cyclic codes over $R$ with reverse and reverse complement constraints. This work can be used to generate general DNA cyclic code over $R$. 


\section{References}

[1] T. Abualrub, R. Oehmke, On the generators of $\mathbb{Z}_{4}$ cyclic codes of length $2^{e}$, IEEE Transactions on Information Theory 49 (2003) 2126-2133.

[2] T. Abualrub, I. Siap, Cyclic codes over the rings $\mathbb{Z}_{2}+u \mathbb{Z}_{2}$ and $\mathbb{Z}_{2}+u \mathbb{Z}_{2}+u^{2} \mathbb{Z}_{2}$, Des Codes Crypt 42 (2007) 273-287.

[3] T. Abualrub, I. Siap, Reversible cyclic codes over $\mathbb{Z}_{4}$, Australasian Journal of Combinatorics 38 (2007) 195-205.

[4] L. M. Adleman, Molecular computation of solutions to combinatorial problems, Science 266 (1994) 1021-1024.

[5] N. Bennenni, K. Guenda, S. Mesnager, New DNA cyclic codes over rings, Adv. Math. Comp. 11(1) (2017) 83-98.

[6] A. Bonnecaze, P. Udaya, Cyclic codes and self-dual codes over $\mathbb{F}_{2}+u \mathbb{F}_{2}$, IEEE Transactions on Information Theory 45 (1999) 1250-1255.

[7] D. Boucher, W. Geiselmann, F. Ulmer, Skew cyclic codes, Applied Algebra in Engineering, Communication and Computing 18 (2007) 379-389.

[8] D. Boucher, F. Ulmer, Coding with skew polynomial rings, Journal of Symbolic Computation 44 (2009) 1644-1656.

[9] Y. Cengellenmis, N. Aydin, A. Dertli, Reversible DNA codes from skew cyclic codes over a ring of order 256, J. Algebra Comb. Discrete Appl. 8(1) (2021) 1-8.

[10] H. Q. Dinh, S. Pattanayak, A. K. Singh, S. Sriboonchitta, Construction of cyclic DNA codes over the $\operatorname{ring} \mathbb{Z}_{4}[u] /\left(u^{2}-1\right)$ based in deletion distance, Theoretical Computer Science 773 (2018) 27-42.

[11] B. Feng, S. S. Bai, B. Y. Chen, X. N. Zhou, The constructions of DNA codes from linear self-dual codes over $Z_{4}$, International Conference on Computer Information Systems and Industrial Applications (CISIA 2015) (2015) 496-498.

[12] K. Guenda, T. A. Gulliver, P. Solé, On cyclic DNA codes, IEEE Inter. Sym. Inform. Theory (2013) 121-125.

[13] A. R. Hammons, V. Kumar, A. R. Calderbank, N. J. A. Sloane, P. Solé, The $\mathbb{Z}_{4}$-linearity of Kerdock, Preparata, Goethals, and related codes, IEEE Transactions on Information Theory 40(2) (1994) 301319.

[14] J. Liang, L. Wang, On cyclic DNA codes over $\mathbb{F}_{2}+u \mathbb{F}_{2}$, Journal of Applied Mathematics and Computing 51 (2015) 81-91.

[15] M. Özen, N. T. Özzaim, N. Aydin, Cyclic codes over $\mathbb{Z}_{4}+u \mathbb{Z}_{4}+u^{2} \mathbb{Z}_{4}$, Turkish Journal of Mathematics 41 (2017) 1235-1247.

[16] A. S. L. Rocha, L. C. B. Faria, J. H. Kleinschmidt, R. Palazzo, M. C. Silva-Filho, DNA sequences generated by $Z_{4}$-linear codes, IEEE International Symposium on Information Theory (2010) 13201324.

[17] I. Siap, T. Abualrub, N. Aydin, P. Seneviratne, Skew cyclic codes of arbitrary length, Int. J. Inform. Coding Theory 2 (2011) 10-20.

[18] B. Yildiz, I. Siap, Cyclic codes over $\frac{\mathbb{F}_{2}[u]}{\left(u^{4}-1\right)}$ and applications to DNA codes, Computers \& Mathematics with Applications 63 (2012) 1169-1176.

[19] S. Zhu, X. Chen, Cyclic DNA codes over $\mathbb{F}_{2}+u \mathbb{F}_{2}+v \mathbb{F}_{2}+u v \mathbb{F}_{2}$ and their applications, J. Appl. Math. Comput. 55 (2017) 479-493. 